\title{
Network Pharmacology-Based Analysis on the Curative Effect of Kunxian Capsules against Rheumatoid Arthritis
}

\author{
Hong-Xia Yan $\mathbb{D}^{1,},{ }^{1,2}$ Chun-Fang Xu $\mathbb{D}^{2},{ }^{2}$ Hong Yang $\left(\mathbb{D},{ }^{2}\right.$ Xiao-Ya Wen $\left(\mathbb{D},{ }^{2,3}\right.$ \\ Zhi-Peng Wang $\mathbb{D},{ }^{2}$ Yan-Hong Chen $\mathbb{D},{ }^{2}$ Jing-Xue Liu $\mathbb{D}^{2},{ }^{2}$ Wan-Sheng Chen $\mathbb{D},{ }^{2,4}$ \\ Shou-Hong Gao $\mathbb{D}^{1,2}$ and Xia Tao $\mathbb{D}^{1,2}$ \\ ${ }^{1}$ College of Traditional Chinese Medicine, Yunnan University of Chinese Medicine, Kunming, Yunnan 650500, China \\ ${ }^{2}$ Department of Pharmacy, Changzheng Hospital, Second Military Medical University, Shanghai 200003, China \\ ${ }^{3}$ College of Chemistry and Bio-Engineering, Yichun University, Yichun, Jiangxi 336000, China \\ ${ }^{4}$ Research and Development Center of Chinese Medicine Resources and Biotechnology, \\ The Ministry of Education (MOE) Key Laboratory for Standardization of Chinese Medicines, \\ Institute of Chinese Materia Medica, Shanghai University of Traditional Chinese Medicine, Shanghai 201203, China \\ Correspondence should be addressed to Wan-Sheng Chen; chenwansheng@smmu.edu.cn, Shou-Hong Gao; \\ gaoshouhong@smmu.edu.cn, and Xia Tao; taoxia2003@126.com
}

Received 12 July 2021; Revised 29 August 2021; Accepted 15 September 2021; Published 30 September 2021

Academic Editor: Talha Bin Emran

Copyright (C) 2021 Hong-Xia Yan et al. This is an open access article distributed under the Creative Commons Attribution License, which permits unrestricted use, distribution, and reproduction in any medium, provided the original work is properly cited.

Kunxian capsules (KCs), a Chinese patent medicine, have been clinically proven to be effective in the treatment of rheumatoid arthritis (RA). However, the chemical profile of $\mathrm{KC}$ remains to be characterized, and the mechanism underlying the protective effect against RA is yet to be elucidated. Here, a network pharmacology-based approach was adopted, integrated with the chemical profiling of KC by UHPLC-Q-TOF/MS. As a result, a total of 67 compounds have been identified from KC extract, among which 43 were authenticated by comparison to the mass spectrum of standard chemicals. ADME behaviors of the chemical constituents of $\mathrm{KC}$ were predicted, resulting in 35 putative active ingredients. Through target prediction of both active ingredients of KC and RA and PPI analysis, core targets were screened out, followed by biological process and related pathway enrichment. Then, a TCM-herb-ingredient-target-pathway network was constructed and a multicomponent, multitarget, and multipathway synergistic mechanism was proposed, providing an information basis for further investigation. The active pharmaceutical ingredients included mainly terpenoids (such as triptolide and celastrol), sesquiterpene pyridines (such as wilforgine and wilforine), and flavonoids (such as icariin, epimedin A, B, and C, and 2"-O-rhamnosylicariside II).

\section{Introduction}

Rheumatoid arthritis (RA), a chronic autoimmune disease, causes progressive articular destruction and associated comorbidities in vascular, metabolic, bone, and psychological domains [1]. The disease is characterized by synovial inflammation and hyperplasia ("swelling"), autoantibody production, cartilage and bone destruction ("deformity"), and systemic features, including cardiovascular, pulmonary, psychological, and skeletal disorders $[1,2]$. With the aggravation of population aging, the incidence of RA has increased from 0.5 to $1 \%$ worldwide. Women are more vulnerable to the disease than men, and those aged 50 to 60 are with relatively higher risk [1].
Currently, the treatment options for RA included non-steroidal anti-inflammatory drugs (NSAIDs), antirheumatic drugs, glucocorticoid, and immunomodulators. However, adverse effects, even drug-induced diseases, were frequently reported with single-target and symptom-based medications, especially with glucocorticoid [3]. Obviously, current one drug-one target-one disease approaches in drug discovery have become increasingly inefficient [4]. Multiple synergistic medications are in great need.

Despite that the origin of RA is unknown, it has become recently evident that RA arises based on genetic and epigenetic factors, with the environment playing an important role [5]. According to the Kyoto Encyclopedia of Genes and 
Genomes (KEGG) database, a large and complex network involving T cells, B cells, macrophages, and various cytokines or chemokines, such as IL-1, IL-6, IL-17, TNF- $\alpha$, and MMPs, participated in the pathogenetic processes of RA. Like cocktail therapy, ethnic herbal medicine tended to provide a multitarget approach against illnesses and ailments.

Kunxian capsules (KCs), belonging to the Class VI New Medications in State Key Task 95 in China, are made from 4 crude drugs, including Tripterygium hypoglaucum (Lévl.) Hutch. (TH), Epimedium brevicornu Maxim. (EB), Cuscuta chinensis Lam. (CC), and Lycium barbarum L. (LB). KC has been demonstrated to have immunomodulatory, cytokineantagonistic, anti-inflammatory, and analgesic effects, without glucocorticoid-like effect to induce the atrophy of immune organs [6]. KC treatment was shown to bring significant mitigating effect in patients with RA, systemic lupus erythematosus, or ankylosing spondylitis [6-10]. However, the pharmacodynamic material basis and the underlying mechanisms of the curative effect of KC remain to be illustrated, largely limiting the global acceptance of KC.

Ultra-high performance liquid chromatography coupled with hybrid triple quadrupole time-of-flight mass spectrometry (UHPLC-Q-TOF/MS) is a high-throughput analytical technology that has rapidly developed in the past decade and is widely used in the fields of environmental science, medicine, drug research, and others [11]. Firstly proposed by Hopkins in 2008 [12], network pharmacology represented an efficient approach to establish relationships between multiple compounds and various targets. Therefore, network pharmacology was thought to be appropriate to investigate the molecular mechanisms of complex systems, such as natural herbs or Traditional Chinese Medicine (TCM) [13]. Based on the network of herbs, targets, and diseases, this strategy could systemically elucidate the mechanisms in a holistic manner, matching the TCM theory intrinsically and providing a new and effective way of the research of Chinese compound formulas [13]. Therefore, the current study aimed to investigate the mechanisms underlying the curative effect of $\mathrm{KC}$ against RA, through a network pharmacology-based approach integrated with chemical characterization by UHPLC-Q-TOF/MS. The schematic workflow is shown in Figure S1.

\section{Materials and Methods}

2.1. Materials and Reagents. Reference substances of hyperoside, triptolide, epimedin C, icariin, and baohuoside I were purchased from the National Institutes for Food and Drug Control (Beijing, China). Arginine, proline, valine, betaine, chlorogenic acid, catechin, L-epicatechin, rutin, isoquercitrin, luteolin-7-O-glucoside, kaempferitrin, astragalin, quercitrin, daidzein, epimedoside A, quercetin, luteolin, epimedin A1, epimedin A, epimedin B, kaempferol, isorhamnetin, icaritin, baohuoside II, sagittatoside A, sagittatoside $\mathrm{B}$, wilforgine, wilforine, $2^{\prime \prime}$-O-rhamnosylicariside II, demethylzeylasteral, celastrol, and baohuoside I were purchased from Dalian Meilun Biotechnology Co. Ltd. (Dalian, China). Epigallocatechin, procyanidin B-3, magnoflorine, wilfortrine, euonine, and wilfornine $\mathrm{A}$ were purchased from Chengdu DeSiTe Biotechnology Co. Ltd. (Chengdu, China). The purity of each compound was determined to be higher than $94 \%$ by HPLC. LC-grade acetonitrile, methanol, and formic acid were purchased from Merck (Darmstadt, Germany). Purified water for experiments was purchased from A.S. Watson Group Ltd. (Hong Kong, China). Kunxian capsules (KCs) were provided by the Guangzhou Chen Li Ji Pharmaceutical Co. Ltd. (Guangzhou, China).

2.2. Preparation of Standard and Sample Solutions. Stock solutions of 43 standards were prepared at a concentration of $1 \mathrm{mg} / \mathrm{mL}$ in methanol. Next, aliquots of each stock solution were mixed and diluted with methanol to achieve a series of standard working solutions. The KC samples were ground to a fine powder. Then powdered $\mathrm{KC}$ of $0.150 \mathrm{~g}$ was suspended with $20 \mathrm{~mL}$ of $70 \%$ methanol-water (v/v) and weighed, and it underwent ultrasonic-assisted extraction $(40 \mathrm{kHz}, 500 \mathrm{w})$ for $15 \mathrm{~min}$, followed by lost weight replenishment. Then, the extracted solution was centrifuged at 15,000 rpm for $20 \mathrm{~min}$, and the supernatant was obtained as sample for detection. All the samples were stored at $4^{\circ} \mathrm{C}$ before analysis.

2.3. UHPLC-Q-TOF/MS Analysis. The LC/MS system consisted of an Agilent 1290 Infinity LC system coupled to an Agilent 6530 UHD accurate-mass quadrupole time-of-flight (Q-TOF) mass spectrometer (Agilent, USA). Chromatographic separations were performed on Accucore C18 $(150 \times 2.1 \mathrm{~mm}, 2.6 \mu \mathrm{m}$, Thermo Fisher Scientific, USA) maintained at $35^{\circ} \mathrm{C}$. The chromatographic conditions were as follows: flow rate of $0.3 \mathrm{~mL} / \mathrm{min}$, sample injection volume of $1 \mu \mathrm{L}$, mobile phase A $(0.05 \%$ formic acid-water $)$, and mobile phase B $(0.05 \%$ formic acid-acetonitrile) with a gradient elution program as follows: $0-3 \mathrm{~min}, 5-12 \% \mathrm{~B}$; 3-10 min, $12-18 \% \mathrm{~B} ; 10-20 \mathrm{~min}, 18-25 \% \mathrm{~B} ; 20-25 \mathrm{~min}$, $25-37 \% \mathrm{~B} ; 25-37 \mathrm{~min}, 37-65 \% \mathrm{~B}$; 37-38 min, 65-95\% B; and 38-40 min, 95\% B.

An electrospray ionization source (ESI) interface was used, and the following parameters were employed in positive modes: capillary voltage, $4.5 \mathrm{kV}$; nebulizer pressure, $50 \mathrm{psi}$; drying gas flow, $11 \mathrm{~L} / \mathrm{min}$; gas temperature, $350^{\circ} \mathrm{C}$; and scan range, $100-1500 \mathrm{~m} / \mathrm{z}$. The collision energy was set at $30 \mathrm{eV}$ to obtain the fragment ion data. Data were collected in centroid mode, and the mass range was set at $\mathrm{m} / \mathrm{z}$ 100-1100 [14-16]. All the acquisition and analysis of data were controlled by Agilent MassHunter Workstation Software Qualitative Analysis Version B.10.0 (Agilent Technologies, USA).

2.4. Identification of the Main Ingredients in KC. The compounds contained in the 4 crude drugs were gathered from multiple databases, including PubMed, SciFinder (scifinder.cas.org/), and TCMSP (tcmspw.com/tcmsp.php), as well as subsequent literature search. A KC-specific in-house library was established, including the basic information of the compounds: name, formula, and exact molecular weight. Agilent MassHunter Workstation for Qualitative Analysis (Version 10.0) was employed to analyze the components of 
KC, which provided a list of compounds that were putatively contained in KC. Chemical standards were obtained and used for the final authentication of the components.

2.5. Target Fishing of $K C$ and $R A$. To screen out putative active ingredient, TCMSP database and SwissADME (swisstargetprediction.ch/) were added to predict the absorption, distribution, metabolism, and excretion (ADME) properties, focusing on 3 aspects, including oral bioavailability (OB), druglikeness (DL), and intestinal permeability. To predict related targets, TCMSP database and SwissTargetPrediction web tool were utilized to gather the potential targets of the screened-out active ingredients. A list of potential targets of the active ingredients of $\mathrm{KC}$ was obtained after duplicate removal. GeneCards database (genecards.org/) was used to predict the related targets to RA. The first 1000 targets with higher relevance score were preserved for further analysis. The overlapping targets between the $\mathrm{KC}$ and RA were extracted using Microsoft Excel and visualized using Venny2.1.0 (https://bioinfogp.cnb.csic.es/tools/venny/index.html).

2.6. PPI Analysis for Core Target Screening. STRING database (string-db.org/) was employed for the PPI analysis with the organism set as "Homo sapiens." The resulting file (.tsv) was introduced to Cytoscape 3.7.0 for topological analysis and visualization. The threshold for core target screening was that the "degree," "betweenness," and "closeness" were all larger than the averages of the whole sample.

2.7. GO and KEGG Pathway Enrichment. After core target screening, $\mathrm{R}$ package from Bioconductor database (bioconductor.org) was adopted to conduct GO and KEGG enrichment based on OmicShare platform (omicshare.com/ tools/index.php/) in 3 modules, including biological process (BP), molecular function (MF), and cellular component (CC). $P<0.05$ and FDR $<0.05$ were used as screening criteria. Cytoscape 3.7.0 software was adopted to visualize the herb-ingredient-target-pathway network of KC, active ingredients, core targets, and key pathways.

\section{Results}

\subsection{Identification of the Chemical Constituents in KC Extract} by UHPLC-Q-TOF/MS. Through UHPLC-Q-TOF/MS analysis, 67 chemical constituents were identified from the $70 \%$ methanol-in-water extract of $\mathrm{KC}$ according to mass fragmentation patterns based on a previously established KC-specified in-house library (Figure 1). Among them, 43 compounds $(1,2,3,4,5,8,10,11,15,16,17,18,19,20,21,22$, $23,25,26,27,28,29,30,31,32,33,34,35,37,43,45,47,48$, $50,51,52,55,58,60,63,64,66$, and 67$)$ were authenticated by comparison to the mass spectrums of the corresponding chemical standards, as indicated by a star symbol $\left({ }^{*}\right)$ in Table S1. The other 24 ingredients were identified by studying on the mass fragmentation patterns through analysis of the mass spectra, as well as by referring to previous reports [17-34]. As a result, 67 compounds were identified in total, including 32 flavonoids, 20 alkaloids (18 sesquiterpene alkaloids), 6 amides, 5 terpenoids, and 4 others.

3.2. Screening of the Potential Active Ingredients. Active ingredients were screened out by analyzing their behaviors of absorption, distribution, metabolism, and excretion (ADME) by referring to TCMSP database and/or prediction using SwissADME web tool. The inclusion of potential active ingredients generally complied with "Rule of Five" (Ro5), firstly proposed by Lipinski et al. in 1997 [35]. Specifically, the thresholds were as follows: for TCMSP database, oral bioavailability $(\mathrm{OB})$ should be no lower than $30 \%$, druglikeness (DL) should be no lower than $0.18 \%$, and intestinal permeability (Caco-2) should be no lower than -0.40 [36]; for SwissADME analysis, gastrointestinal absorption (GI) should be indicated as "high," and DL analysis should result in more than 2 "Yes," with data shown in Table S2 (In total, 5 filters of rule-of-five were adopted in the study, including Lipinski (Pfizer), Ghose (Amgen), Veber (GSK), Egan (Pharmacia,) and Muegge (Bayer) methods. One "Yes" was counted if no violations were found in one filter. The times of found "Yes"s were presented as results [37] (see swissADME.ch.)). Moreover, notwithstanding their predicted compromised ADME behaviors, some other compounds with reported strong pharmacological activities were also preserved for further analysis, such as epimedin A [38, 39], 2" -O-rhamnosylicariside II (rha-icariside) [40], and wilfornine A [23]. Finally, 35 active ingredients stood out as presented in Table 1 and Figure 2, including 19 flavonoids (3 flavones, 4 flavonols, and 3 flavanes), 10 alkaloids (8 sesquiterpene pyridine alkaloids), 4 terpenoids, and 2 amino acids.

3.3. Target Fishing and Major Hub Analysis. Target fishing was conducted for the 35 candidate compounds by searching the PubChem and TCMSP databases or by virtual prediction through SwissTargetPrediction web tool. UniProt Consortium was employed to convert the targets to uniform gene symbol. Finally, merging and duplicate removal yielded 874 potential targets for the 35 active ingredients in KC (Table S3). Using GeneCards database, the first 1000 relevant targets for RA were obtained (Table S4). Then, 228 overlapping targets (Table S5) between KC and RA (Figure 3(a)) were introduced into STRING database for PPI analysis. A PPI network with 228 nodes and 14356 edges (Figure 3(b)) was constructed. Subsequent topological analysis by Cytoscape software presented 51 major hubs that might play a potential important role in the curative effect of KC for RA (Table S6).

3.4. GO and KEGG Enrichment. GO enrichment of the core targets indicated that multiple biological processes (BP), cellular components (CC), and molecular functions (MF) have been engaged in the mechanisms underlying the protective effect of KC against RA (Figure 4(a)). The top 10 enriched BP included biological regulation, cellular process, developmental process, multicellular organismal process, positive regulation of biological process, regulation of 


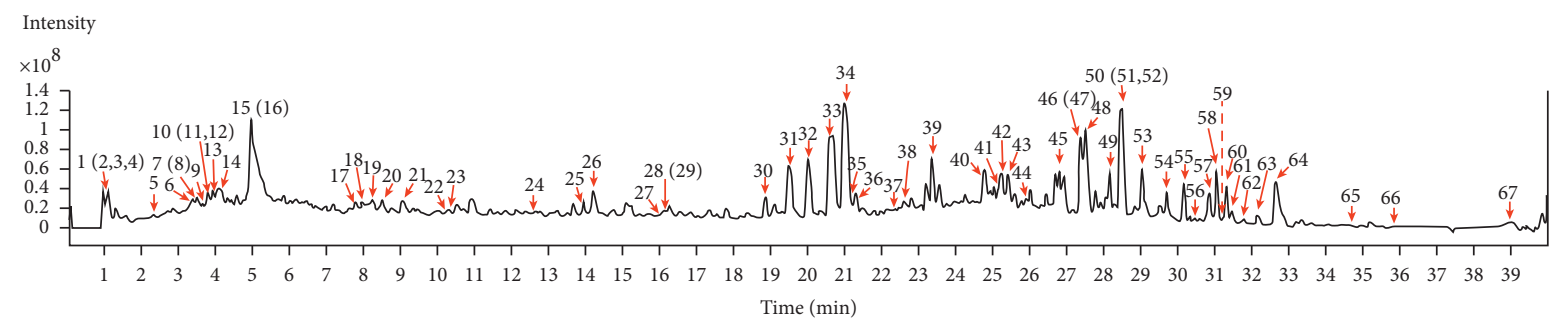

FIgURE 1: Representative total ion current (TIC) chromatogram of KC extract by UHPLC-Q-TOF/MS in positive mode.

TABLE 1: The 35 putative active ingredients in KC.

\begin{tabular}{|c|c|c|c|c|}
\hline Peak no. & Name & Formula & CAS & Sources \\
\hline 2 & Proline & $\mathrm{C}_{5} \mathrm{H}_{9} \mathrm{NO}_{2}$ & $344-25-2$ & $\mathrm{CC}, \mathrm{LB}$ \\
\hline 3 & Valine & $\mathrm{C}_{5} \mathrm{H}_{11} \mathrm{NO}_{2}$ & $72-18-4$ & CC, LB \\
\hline 4 & Betaine & $\mathrm{C}_{5} \mathrm{H}_{11} \mathrm{NO}_{2}$ & $107-43-7$ & LB \\
\hline 5 & Epigallocatechin & $\mathrm{C}_{15} \mathrm{H}_{14 \mathrm{a}} \mathrm{O}_{7}$ & $970-74-1$ & TH \\
\hline 11 & Catechin & $\mathrm{C}_{15} \mathrm{H}_{14} \mathrm{O}_{6}$ & $7295-85-4$ & $\mathrm{TH}, \mathrm{CC}$ \\
\hline 15 & Magnoflorine & $\mathrm{C}_{20} \mathrm{H}_{23} \mathrm{NO}_{4}$ & $2141-09-5$ & EB \\
\hline 16 & L-Epicatechin & $\mathrm{C}_{15} \mathrm{H}_{14} \mathrm{O}_{6}$ & $490-46-0$ & $\mathrm{TH}, \mathrm{CC}$ \\
\hline 18 & Hyperoside & $\mathrm{C}_{21} \mathrm{H}_{20} \mathrm{O}_{12}$ & 482-36-0 & $\mathrm{EB}, \mathrm{CC}$ \\
\hline 24 & Baohuosu & $\mathrm{C}_{22} \mathrm{H}_{22} \mathrm{O}_{7}$ & $119730-90-4$ & $\mathrm{~EB}$ \\
\hline 25 & Daidzein & $\mathrm{C}_{15} \mathrm{H}_{10} \mathrm{O}_{4}$ & 486-66-8 & $\mathrm{EB}, \mathrm{CC}$ \\
\hline 27 & Triptolide & $\mathrm{C}_{20} \mathrm{H}_{24} \mathrm{O}_{6}$ & $38748-32-2$ & $\mathrm{TH}$ \\
\hline 28 & Quercetin & $\mathrm{C}_{15} \mathrm{H}_{10} \mathrm{O}_{7}$ & $117-39-5$ & EB \\
\hline 29 & Luteolin & $\mathrm{C}_{15} \mathrm{H}_{10} \mathrm{O}_{6}$ & 491-70-3 & $\mathrm{EB}, \mathrm{CC}$ \\
\hline 31 & Epimedin A & $\mathrm{C}_{39} \mathrm{H}_{50} \mathrm{O}_{20}$ & $140147-77-9$ & EB \\
\hline 32 & Epimedin B & $\mathrm{C}_{38} \mathrm{H}_{48} \mathrm{O}_{19}$ & $110623-73-9$ & $\mathrm{~EB}$ \\
\hline 33 & Epimedin C & $\mathrm{C}_{39} \mathrm{H}_{50} \mathrm{O}_{19}$ & $110642-44-9$ & $\mathrm{~EB}$ \\
\hline 34 & Icariin & $\mathrm{C}_{33} \mathrm{H}_{40} \mathrm{O}_{15}$ & 489-32-7 & EB \\
\hline 35 & Kaempferol & $\mathrm{C}_{15} \mathrm{H}_{10} \mathrm{O}_{6}$ & $520-18-3$ & $\mathrm{CC}$ \\
\hline 37 & Isorhamnetin & $\mathrm{C}_{16} \mathrm{H}_{12} \mathrm{O}_{7}$ & $480-19-3$ & $\mathrm{CC}$ \\
\hline 43 & Baohuoside II/ikarisoside A & $\mathrm{C}_{26} \mathrm{H}_{28} \mathrm{O}_{10}$ & $55395-07-8$ & $\mathrm{~EB}$ \\
\hline 46 & Tripterifordin & $\mathrm{C}_{20} \mathrm{H}_{30} \mathrm{O}_{3}$ & $139122-81-9$ & TH \\
\hline 47 & Sagittatoside B & $\mathrm{C}_{32} \mathrm{H}_{38} \mathrm{O}_{14}$ & $118525-36-3$ & $\mathrm{~EB}$ \\
\hline 48 & $2^{\prime \prime}$-O-Rhamnosylicariside II (rha-icariside) & $\mathrm{C}_{33} \mathrm{H}_{40} \mathrm{O}_{14}$ & $135293-13-9$ & $\mathrm{~EB}$ \\
\hline 50 & Icaritin & $\mathrm{C}_{21} \mathrm{H}_{20} \mathrm{O}_{6}$ & $118525-40-9$ & EB \\
\hline 51 & Baohuoside I/icarisid & $\mathrm{C}_{27} \mathrm{H}_{30} \mathrm{O}_{10}$ & $113558-15-9$ & $\mathrm{~EB}$ \\
\hline 52 & Wilfortrine & $\mathrm{C}_{41} \mathrm{H}_{47} \mathrm{NO}_{20}$ & $37239-48-8$ & TH \\
\hline 53 & Peritassine A & $\mathrm{C}_{38} \mathrm{H}_{47} \mathrm{NO}_{18}$ & $150881-01-9$ & TH \\
\hline 55 & Wilfordine & $\mathrm{C}_{43} \mathrm{H}_{49} \mathrm{NO}_{19}$ & $37239-51-3$ & $\mathrm{TH}$ \\
\hline 57 & Wilfornine D & $\mathrm{C}_{43} \mathrm{H}_{49} \mathrm{NO}_{21}$ & NA & $\mathrm{TH}$ \\
\hline 58 & Wilforgine & $\mathrm{C}_{41} \mathrm{H}_{47} \mathrm{NO}_{19}$ & $37239-47-7$ & $\mathrm{TH}$ \\
\hline 59 & Triptophenolide & $\mathrm{C}_{20} \mathrm{H}_{24} \mathrm{O}_{3}$ & $74285-86-2$ & $\mathrm{TH}$ \\
\hline 60 & Euonine & $\mathrm{C}_{38} \mathrm{H}_{47} \mathrm{NO}_{18}$ & $41758-69-4$ & $\mathrm{TH}$ \\
\hline 63 & Wilfornine A & $\mathrm{C}_{45} \mathrm{H}_{51} \mathrm{NO}_{20}$ & $345954-00-9$ & $\mathrm{TH}$ \\
\hline 64 & Wilforine & $\mathrm{C}_{43} \mathrm{H}_{49} \mathrm{NO}_{18}$ & 11088-09-8 & $\mathrm{TH}$ \\
\hline 67 & Celastrol & $\mathrm{C}_{29} \mathrm{H}_{38} \mathrm{O}_{4}$ & $34157-83-0$ & $\mathrm{TH}$ \\
\hline
\end{tabular}

Note. TH, Tripterygium hypoglaucum (Lévl.) Hutch.; EB, Epimedium brevicornu Maxim.; CC, Cuscuta chinensis Lam.; LB, Lycium barbarum L.

biological process, response to stimulus, signaling, metabolic process, and cellular component organization or biogenesis. The top 10 related CC included cell, cell part, organelle, organelle part, membrane, protein-containing complex, membrane-enclosed lumen, extracellular region, membrane part, and extracellular region part. The top 3 related MF included binding, catalytic activity, and molecular function regulator. After pathway enrichment using KEGG, the top 10 relevant pathways are shown in Figure 4(b), including PI3K-Akt signaling pathway, TNF signaling pathway, T cell receptor signaling pathway, IL-17 signaling pathway, JAKSTAT signaling pathway, MAPK signaling pathway, Toll-like receptor signaling pathway, Th17 cell differentiation, and VEGF signaling pathway.

3.5. Construction of TCM-Herb-Ingredient-Target-Pathway Network. Through literature research, we linked up the aforementioned 10 pathways, key targets, and active ingredients, constructed a TCM-herb-ingredient-target-pathway 


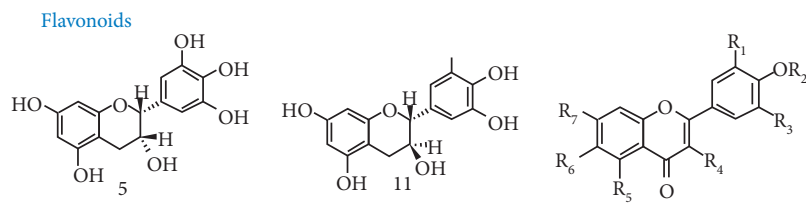<smiles>Oc1cc(O)c2c(c1)O[C@H](c1ccc(O)c(O)c1)[C@H]2O</smiles>
16<smiles>COc1cc(-c2cc(=O)c3c(O)cc(O)c(CCC(C)C)c3o2)cc(OC)c1O</smiles>

$18: \mathrm{R}_{1}=\mathrm{OH}, \mathrm{R}_{2}=\mathrm{H}, \mathrm{R}_{3}=\mathrm{H}, \mathrm{R}_{4}=\mathrm{Glc}, \mathrm{R}_{5}=\mathrm{OH}, \mathrm{R}_{6}=\mathrm{H}, \mathrm{R}_{7}=\mathrm{OH}$ 25: $\mathrm{R}_{1}=\mathrm{H}, \mathrm{R}_{2}=\mathrm{H}, \mathrm{R}_{3}=\mathrm{H}, \mathrm{R}_{4}=\mathrm{H}, \mathrm{R}_{5}=\mathrm{OH}, \mathrm{R}_{6}=\mathrm{OH}, \mathrm{R}_{7}=\mathrm{H}$ $28: \mathrm{R}_{1}=\mathrm{H}, \mathrm{R}_{2}=\mathrm{H}, \mathrm{R}_{3}=\mathrm{OH}, \mathrm{R}_{4}=\mathrm{OH}, \mathrm{R}_{5}=\mathrm{OH}, \mathrm{R}_{6}=\mathrm{H}, \mathrm{R}_{7}=\mathrm{OH}$ 29: $\mathrm{R}_{1}=\mathrm{H}, \mathrm{R}_{2}=\mathrm{H}, \mathrm{R}_{3}=\mathrm{OH}, \mathrm{R}_{4}=\mathrm{H}, \mathrm{R}_{5}=\mathrm{OH}, \mathrm{R}_{6}=\mathrm{H}, \mathrm{R}_{7}=\mathrm{OH}$ 35: $\mathrm{R}_{1}=\mathrm{H}, \mathrm{R}_{2}=\mathrm{H}, \mathrm{R}_{3}=\mathrm{H}, \mathrm{R}_{4}=\mathrm{OH}, \mathrm{R}_{5}=\mathrm{OH}, \mathrm{R}_{6}=\mathrm{H}, \mathrm{R}_{7}=\mathrm{OH}$ $37: \mathrm{R}_{1}=\mathrm{H}, \mathrm{R}_{2}=\mathrm{H}, \mathrm{R}_{3}=\mathrm{OCH}_{3}, \mathrm{R}_{4}=\mathrm{OH}, \mathrm{R}_{5}=\mathrm{OH}, \mathrm{R}_{6}=\mathrm{H}, \mathrm{R}_{7}=\mathrm{OH}$<smiles>COc1ccc(-c2c3oc4c(CC=C(C)C)c(O)cc(O)c4oc-3c(O)c2=O)cc1</smiles>

31: $\mathrm{R}_{1}=\mathrm{Glc}-(1 \rightarrow 2)-\mathrm{Rha}, \mathrm{R}_{2}=\mathrm{Glc}, \mathrm{R}_{3}=\mathrm{CH}$ 32: $\mathrm{R}_{1}=\mathrm{Xyl}-(1 \rightarrow 2)-\mathrm{Rha}, \mathrm{R}_{2}=\mathrm{H}, \mathrm{R}_{3}=\mathrm{CH}_{3}$ 33: $\mathrm{R}_{1}=$ Rha- $(1 \rightarrow 2)-$ Rha, $\mathrm{R}_{2}=\mathrm{Glc}, \mathrm{R}_{3}=\mathrm{CH}_{3}$ 34: $\mathrm{R}_{1}=\mathrm{Rha}, \mathrm{R}_{2}=\mathrm{Glc}, \mathrm{R}_{3}=\mathrm{CH}_{3}$

43: $\mathrm{R}_{1}=\mathrm{Rha}, \mathrm{R}_{2}=\mathrm{H}, \mathrm{R}_{3}=\mathrm{H}$

$47: \mathrm{R}_{1}=\mathrm{Xyl}-(1 \rightarrow 2)-\mathrm{Rha}, \mathrm{R}_{2}=\mathrm{H}, \mathrm{R}_{3}=\mathrm{CH}_{3}$ 48: $\mathrm{R}_{1}=$ Rha- $(1 \rightarrow 2)-\mathrm{Rha}, \mathrm{R}_{2}=\mathrm{H}, \mathrm{R}_{3}=\mathrm{CH}_{3}$ 51: $\mathrm{R}_{1}=\mathrm{H}, \mathrm{R}_{2}=\mathrm{H}, \mathrm{R}_{3}=\mathrm{H}$ 51: $\mathrm{R}_{1}=\mathrm{Rha}, \mathrm{R}_{2}=\mathrm{H}, \mathrm{R}_{3}=\mathrm{CH}_{3}$

Alkaloids

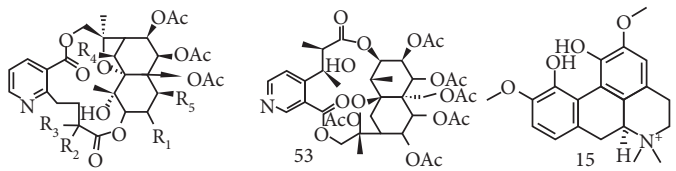

52: $\mathrm{R}_{1}=\mathrm{O}, \mathrm{R}_{2}=\mathrm{OH}, \mathrm{R}_{3}=\mathrm{CH}_{3}, \mathrm{R}_{4}=\mathrm{OAc}, \mathrm{R}_{5}=\mathrm{OAc}$ 55: $\mathrm{R}_{1}=\mathrm{Z}_{-3}, \mathrm{R}_{2}=\mathrm{OH}, \mathrm{R}_{3}=\mathrm{CH}_{3}, \mathrm{R}_{4}=\mathrm{OAc}, \mathrm{R}_{5}=\mathrm{OAc}$ 57: $\mathrm{R}_{1}=\mathrm{OAc}, \mathrm{R}_{2}=\mathrm{CH}_{3}, \mathrm{R}_{3}=\mathrm{O}_{\mathbf{2}} \mathrm{O} \mathrm{CO}_{4}, \mathrm{R}_{4}=\mathrm{OAc}, \mathrm{R}_{5}=\mathrm{OAc}$ 58: $\mathrm{R}_{1}=\mathrm{O}_{3} \mathrm{O}, \mathrm{R}_{2}=\mathrm{CH}_{3}, \mathrm{R}_{3}=\mathrm{H}, \mathrm{R}_{4}=\mathrm{OAc}, \mathrm{R}_{5}=\mathrm{OAc}$<smiles>C[N+](C)(C)CC(=O)O</smiles>
60: $\mathrm{R}_{1}=\mathrm{OAc}, \mathrm{R}_{2}=\mathrm{CH}_{3}, \mathrm{R}_{3}=\mathrm{H}, \mathrm{R}_{4}=\mathrm{OAc}, \mathrm{R}_{5}=\mathrm{OAc}$ 63: $\mathrm{R}_{1}=\mathrm{OAc}, \mathrm{R}_{2}=\mathrm{CH}_{3}, \mathrm{R}_{3}=\mathrm{O}_{-} \mathrm{O}, \mathrm{R}_{4}=\mathrm{OAc}, \mathrm{R}_{5}=\mathrm{OAc}$ 64: $\mathrm{R}_{-\xi \xi} \mathrm{O}-\mathrm{C}_{\mathrm{O}}, \mathrm{R}_{2}=\mathrm{CH}_{3}, \mathrm{R}_{3}=\mathrm{H}, \mathrm{R}_{4}=\mathrm{OAc}, \mathrm{R}_{5}=\mathrm{OAc}$

Terpenoids

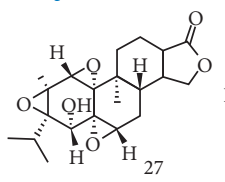

4

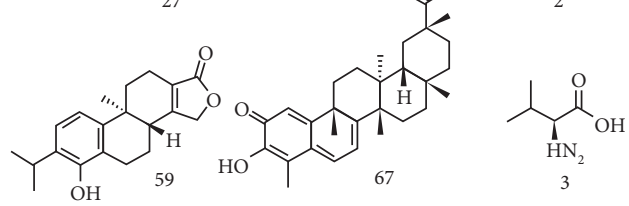

Figure 2: The structures of the 35 potentially active ingredients in KC.

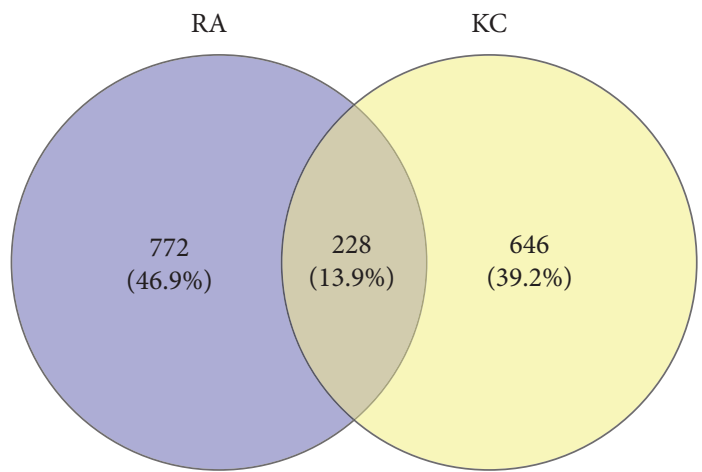

(a)

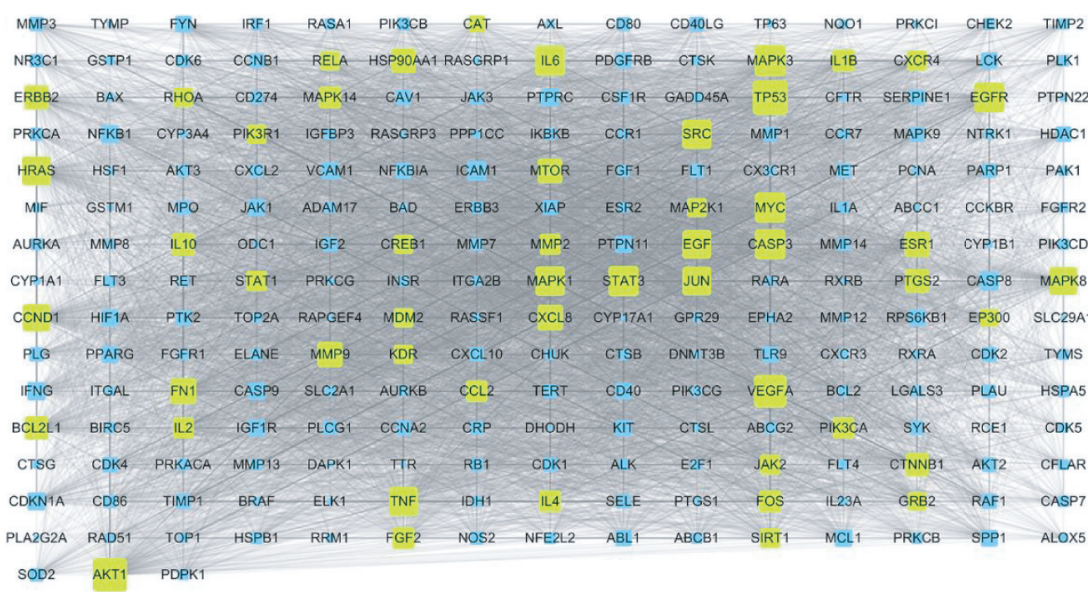

(b)

Figure 3: Prediction of key targets. Venn diagram (a) and the PPI network (b). 


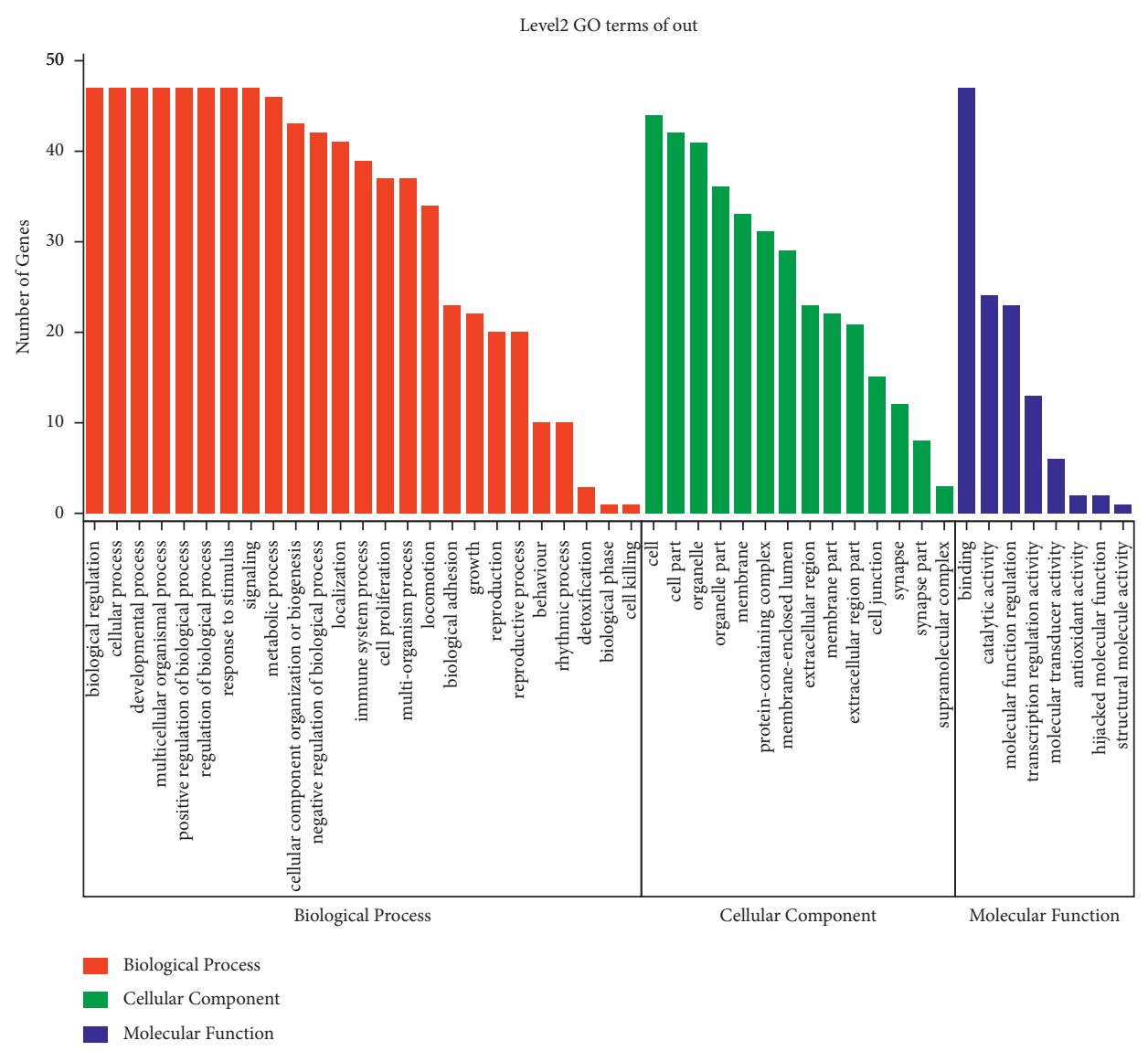

(a)

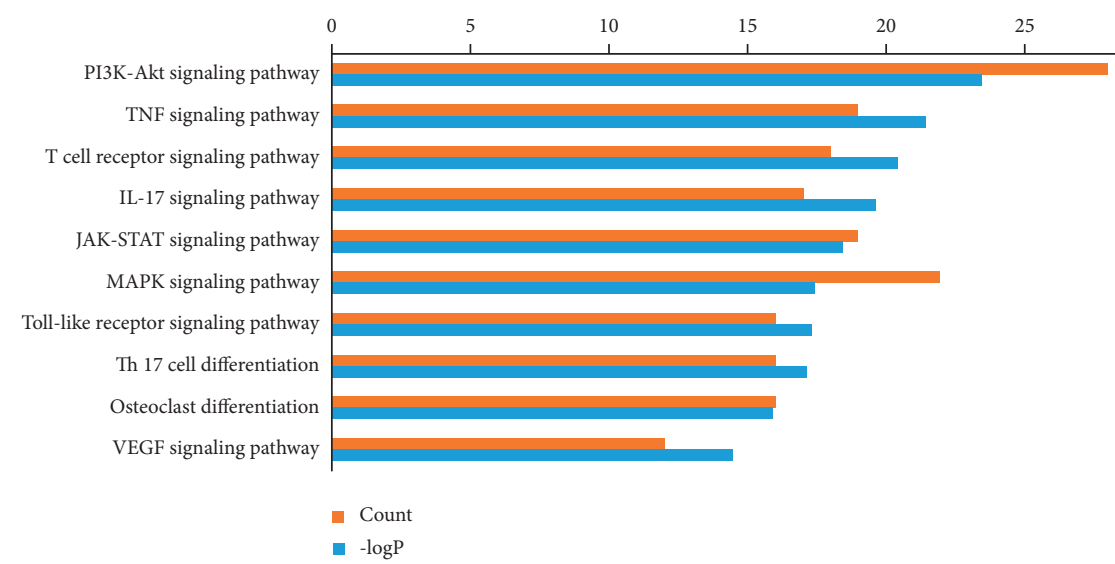

(b)

Figure 4: GO and KEGG enrichment. (a) The relevant biological processes, cellular components, and molecular functions. (b) The top 10 related pathways.

network (Figure 5), and proposed a possible mechanism underlying the curative effect of $\mathrm{KC}$ against RA (Figure 6). The network contained 95 nodes and 537 edges, with specific information shown in Table s7.

\section{Discussion}

The current work investigated the potential mechanisms of the curative effect of $\mathrm{KC}$, a Chinese patent medicine, against RA, by integrating the chemical profiling with network pharmacology analysis, for the first time [6, 41]. The establishment of TCM-herb-ingredient-target-pathway network could make a useful reference for further drug development and the treatment of RA. The efficacy of KC against RA has been proved by multicenter clinical trials [6-10]. By analyzing open-access databases, Tang et al. collected related information of $\mathrm{KC}$ and constructed a disease network for the protection of KC against RA [6], whereas no chemical characterization was conducted. Jing et al. investigated the potential mechanism of $\mathrm{KC}$ against 


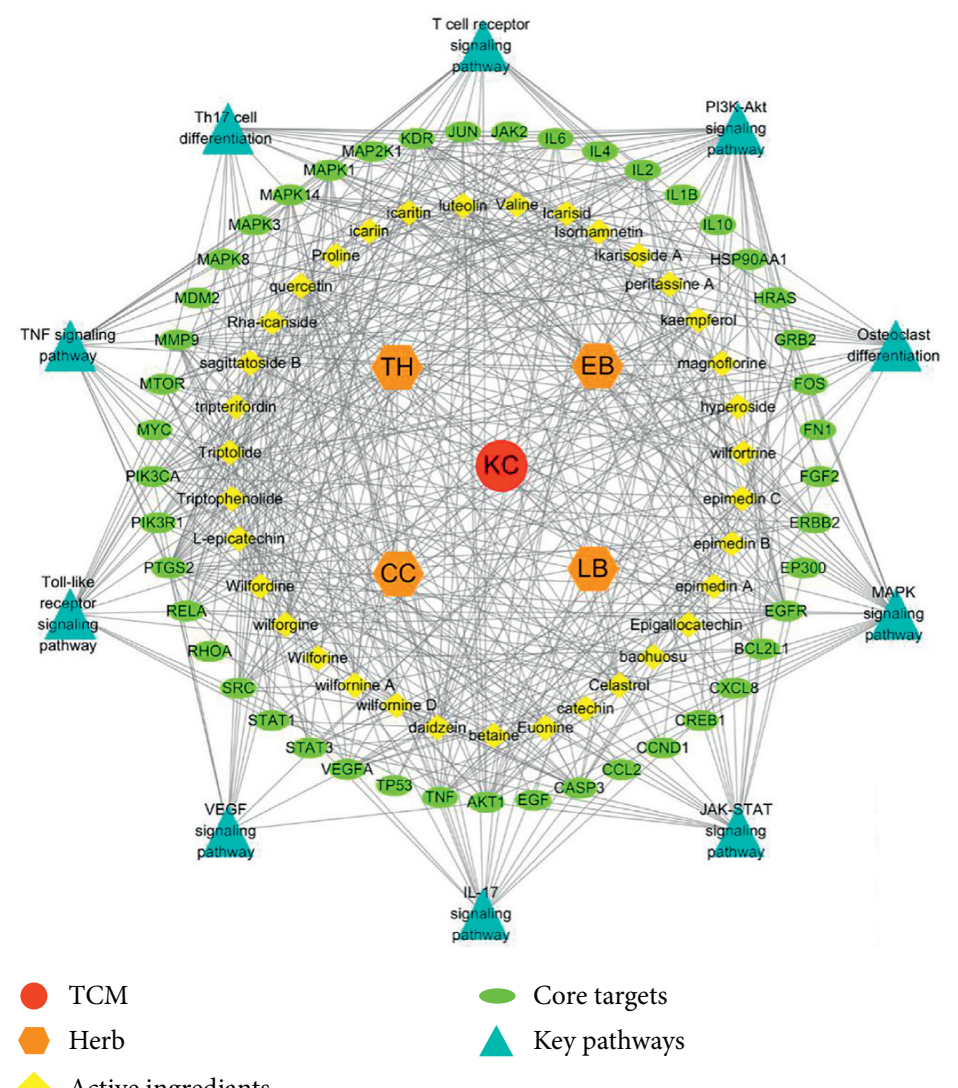

FIGURE 5: Construction of TCM-herb-ingredient-target-pathway network.

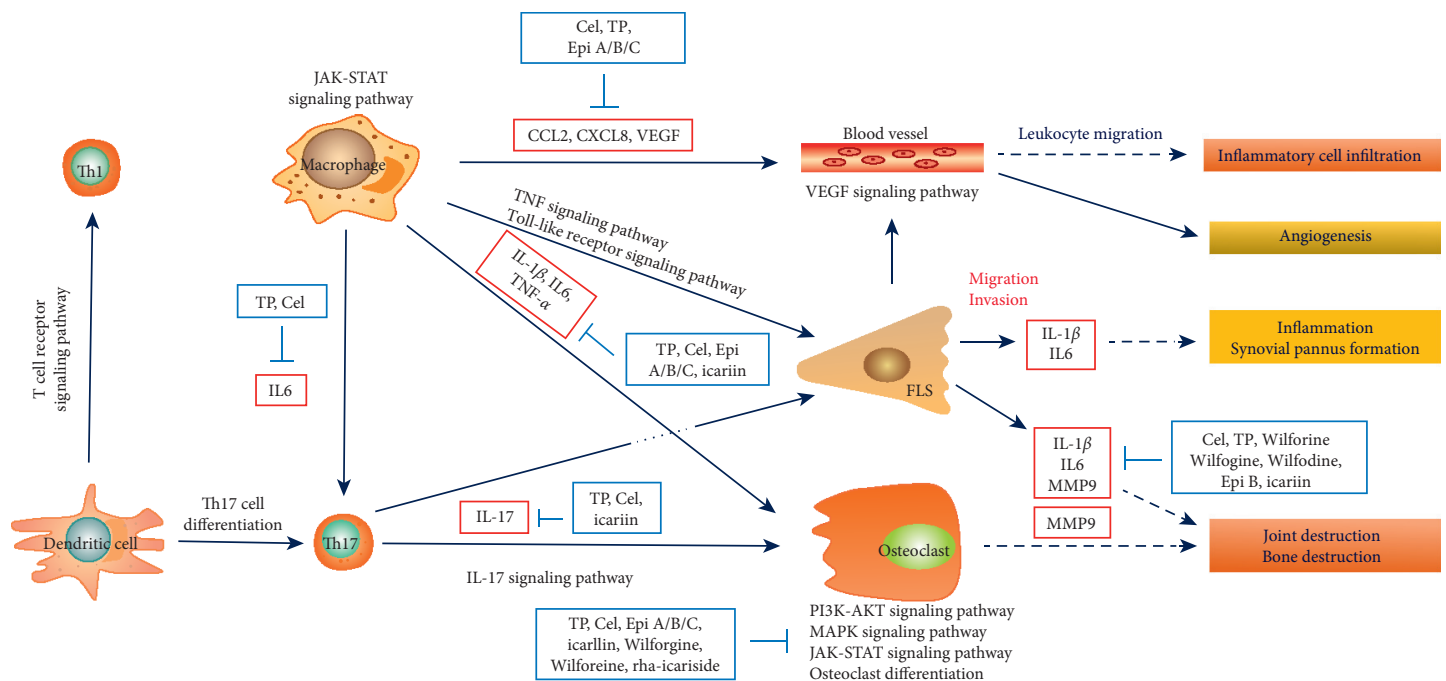

Figure 6: The potential mechanism underlying the protective effect of KC against RA. Note: Cel, celastrol; TP, triptolide; epi A/B/C, epimedin A/B/C; rha-icariside, $2^{\prime \prime}$-O-rhamnosylicariside II.

proteinuria and identified 51 chemical compounds from $\mathrm{KC}$, among which 9 compounds were compared with corresponding reference standards [41]. Instead, our study identified 67 compounds from $\mathrm{KC}$ extract, and 43 components were authenticated by reference standard comparison.

The pharmacodynamic material basis was investigated in our study. Based on predicted ADME behaviors and previous reports, 35 potentially active ingredients were screened out. Among those compounds, 8 sesquiterpene pyridine alkaloids $(52,53,55,57,58,60,63$, and 64$)$ were demonstrated to have immunosuppressive effect [23, 42], which originated from $\mathrm{TH}$, one of the principal herbs in $\mathrm{KC}$. As the principle sesquiterpene pyridines, wilforgine (58) and wilforine (64) were reported to exhibit immunosuppressive 
and anti-inflammatory effects [42]. Besides, 2 terpenoids, triptolide (27) and celastrol (67), seemed to be worth our attention. Triptolide was reported to suppress activation of inflammation, thus inhibiting bone loss (osteopenia) [43]. Fan et al. investigated the potential mechanism underlying which triptolide exerted therapeutic effect against RA, by combining bioinformatics analysis with experiment validation [44]. They highlighted that triptolide could inhibit inflammatory responses in RA by triggering receptors expressed on myeloid cells related to (TREM)-1 signaling pathway [44]. Moreover, it was found that in vitro and in vivo, triptolide treatment significantly reduced the migratory and invasive capacities of fibrolast-like synoviocytes (FLS), which would have tumor-like features when activated [45]. FLS, a major constituent of the synovial hyperplasia, could play a pivotal role in RA invading cartilage and bone tissue [46-48]. The related effector molecules to FLS, including inflammatory factors and MAPKs, have also emerged as important mediators of RA [46]. Celastrol, also known as tripterine, also has various pharmacological functions. Li et al. [49] demonstrated that tripterine could protect a chondrogenic cell line ATDC5 from lipopolysaccharide (LPS) injury in vitro. Besides, celastrol induced autophagy-dependent cytotoxicity in synovial fibroblasts (the primary form of FLS) and repressed arthritis in vivo, possibly through blockage of calcium signaling [50]. The other principal herb, EB, provided 15 putative active ingredients, including 14 flavonoids. Icariin (34), one of the main flavonoids, was shown to alleviate RA in a murine model [51] and to inhibit proliferation and inflammation, promoting apoptosis of FLS [52]. Besides, 3 flavonoids, epimedin A (31), B (32), and C (33), and 2" -O-rhamnosylicariside II (rha-icariside, 48) showed the most profound anabolic and anti-inflammatory effects on human osteoarthritic chondrocytes $[38,39,53]$.

As depicted in KEGG database and Figures 4(b)-6, the pathogenesis of RA might involve multiple immune and inflammatory cells, such as dendric cells (DC), T cells, macrophages, and effector cells, including chondrocytes, osteoclast, FLS, and vessel cells. Several signaling pathways were supposed to be involved in the process. T cell receptor signaling pathway participated in the differentiation of selfreactive Th1 cell from DC. The differentiation of Th17 cell and the secretion of IL-17 were supposed to be the early activator of T cell-mediated inflammatory responses. These three pathways represented early promoter of the occurrence of RA. Then, signaling pathways of JAK-STAT, PI3K-AKT, and MAPK were involved in the osteoclast differentiation, thus leading to bone and joint destruction. FLS could be activated through TNF signaling pathway and Toll-like receptor signaling pathway, thereby promoting the inflammation. Besides, VEGF signaling pathway could be involved in the leukocyte migration and inflammatory cell infiltration.

It was suggested that KC exerted its efficacy against RA through a complex network that involved various components, multiple pathways, and many targets. First and foremost, KC could directly intervene the enhanced differentiation and activation of osteoclasts, thus ameliorating local bone erosion and systemic osteoporosis in RA [54]. Triptolide has been shown to inhibit the phosphorylation of PI3K and AKT in bone marrow mononuclear cells [43], to decrease the phosphorylation of JAK and STAT in the human leukemic U937 cells and in collagen-induced arthritis rats [44], and to inhibit the activation of matrix metalloproteinase 9 (MMP-9). Moreover, KC could inhibit the excessive inflammatory response and regulate the immune reaction [6]. Icariin inhibited STAT3 activation in T cells, resulting in decreased IL-17 production and alleviated RA [51]. Moreover, IL-6, IL-1 $\beta$, and TNF- $\alpha$ were vital pathogenicity cytokines, which could be reduced by components of TH $[52,53,55]$. Other involved chemokines included CCLs and CXCLs [38]. Other than those targets, VEGF was involved in the generation of pannus, an inflammatory exudate overlying synovial cells commonly occurring in RA.

\section{Conclusions}

In conclusion, KC possibly attenuated RA through a multicomponent, multitarget, and multipathway synergistic mechanism. It was suggested that the pharmacodynamic material basis included terpenoids and sesquiterpene pyridines from Tripterygium hypoglaucum, such as triptolide, celastrol, wilforgine, and wilforine, and flavonoids from Epimedium brevicornu, such as icariin, epimedin A, B, and C, and 2"-O-rhamnosylicariside II. At last, further in-depth studies are still needed to verify the proposed pharmacodynamic material basis and related pathways.

\section{Abbreviations}

NSAIDs: Non-steroidal anti-inflammatory drugs

KC: $\quad$ Kunxian capsule

RA: $\quad$ Rheumatoid arthritis

TCM: $\quad$ Traditional Chinese Medicine

TH: $\quad$ Tripterygium hypoglaucum (Lévl.) Hutch.

EB: $\quad$ Epimedium brevicornu Maxim.

CC: $\quad$ Cuscuta chinensis Lam.

LB: $\quad$ Lycium barbarum L.

TIC: $\quad$ Total ion current

ESI: $\quad$ Electrospray ionization mass spectrometry

TCMSP: $\quad$ Traditional Chinese Medicine Systems Pharmacology Database and Analysis Platform

ADME: Absorption, distribution, metabolism, and excretion

PPI: $\quad$ Protein-protein interaction

GO: $\quad$ Gene Ontology

KEGG: $\quad$ Kyoto Encyclopedia of Genes and Genomes

FDR: $\quad$ False discovery rate

OB: $\quad$ Oral bioavailability

DL: $\quad$ Druglikeness

GI: $\quad$ Gastrointestinal

CAS: $\quad$ Chinese Academy of Sciences

Cel: $\quad$ Celastrol

TP: $\quad$ Triptolide

epi A/B/C: Epimedin A/B/C

rha-icariside: $2^{\prime \prime}$-O-Rhamnosylicariside II. 


\section{Data Availability}

The data used to support the findings of this study are available from the corresponding author upon request.

\section{Conflicts of Interest}

The authors declare that they have no conflicts of interest.

\section{Authors' Contributions}

Xia Tao, Shou-Hong Gao, and Wan-Sheng Chen were responsible for the concept, design, literature search, and manuscript review. Chun-Fang $\mathrm{Xu}$ and Hong Yang made contributions to target prediction, result analysis, and manuscript drafting. Hong-Xia Yan, Xiao-Ya Wen, and Jing-Xue Liu were responsible for the lab work, network pharmacological analysis, and drafting the manuscript. ZhiPeng Wang and Yan-Hong Chen reviewed the data analysis and first draft. All authors have read and approved the manuscript. Hong-Xia Yan, Chun-Fang Xu, Hong Yang, and Xiao-Ya Wen contributed equally to this work.

\section{Acknowledgments}

This study was supported by the National Key R\&D Program of China (no. 2018YFC1707304).

\section{Supplementary Materials}

Figure S1: graphical abstract. Table S1: peak information of the TIC chromatogram. Table S2: the predicted ADME behaviors of the 67 ingredients in KC. Table S3: target prediction of the putative active ingredients. Table S4: target prediction of RA. Table S5: overlapping of the predicted targets of ingredients and RA. Table S6: the key targets and their topological parameters by PPI. Table S7: nodes and edges in the TCM-herb-ingredient-target-pathway network. (Supplementary Materials)

\section{References}

[1] D. Aletaha and J. S. Smolen, "Diagnosis and management of rheumatoid arthritis," Journal of the American Medical Association, vol. 320, no. 13, pp. 1360-1372, 2018.

[2] I. B. McInnes and G. Schett, "Pathogenetic insights from the treatment of rheumatoid arthritis," The Lancet, vol. 389, no. 10086, pp. 2328-2337, 2017.

[3] G. R. Burmester and J. E. Pope, "Novel treatment strategies in rheumatoid arthritis," The Lancet, vol. 389, no. 10086, pp. 2338-2348, 2017.

[4] A. I. Casas, A. A. Hassan, S. J. Larsen et al., "From single drug targets to synergistic network pharmacology in ischemic stroke," Proceedings of the National Academy of Sciences, vol. 116, no. 14, pp. 7129-7136, 2019.

[5] H. U. Scherer, T. Häupl, and G. R. Burmester, "The etiology of rheumatoid arthritis," Journal of Autoimmunity, vol. 110, p. 102400, 2020.

[6] Y. Tang, Y. Zhang, L. Li, Z. Xie, C. Wen, and L. Huang, "Kunxian capsule for rheumatoid arthritis: inhibition of inflammatory network and reducing adverse reactions through drug matching," Frontiers in Pharmacology, vol. 11, p. 485, 2020.

[7] Y. F. Liu, Z. Zhang, J. J. Zhang, Z. Chen, S. H. Tu, and G. L. Xing, "The derivative of tripterygium wilfordii hook F-kunxian capsule, attenuated rheumatoid arthritis: a systematic review and meta-analysis," Evidence-Based Complementary and Alternative Medicine: eCAM, vol. 2020, p. $4178140,2020$.

[8] Y. Liu, Y. Lei, B. Zhang, L. Xia, J. Lu, and H. Shen, "Comparison of Kunxian with leflunomide in the treatment of rheumatoid arthritis," Progress of Anatomical Sciences, vol. 25, no. 1, pp. 17-20, 2019.

[9] C. S. Lin, X. Y. Yang, and L. Dai, "Multi-center clinical study on therapeutic effect of Kunxian capsule on rheumatoid arthritis," Zhongguo Zhong Xi Yi Jie He Za Zhi, vol. 31, no. 6, pp. 769-774, 2011.

[10] Q. Li, L. Li, L. Bi et al., "Kunxian capsules in the treatment of patients with ankylosing spondylitis: a randomized placebocontrolled clinical trial," Trials, vol. 17, no. 1, p. 337, 2016.

[11] Y. Wang, C. Li, L. Li et al., "Application of UHPLC-Q/TOFMS-based metabolomics in the evaluation of metabolites and taste quality of Chinese fish sauce (Yu-lu) during fermentation," Food Chemistry, vol. 296, pp. 132-141, 2019.

[12] A. L. Hopkins, "Network pharmacology: the next paradigm in drug discovery," Nature Chemical Biology, vol. 4, no. 11, pp. 682-690, 2008.

[13] R. Zhang, X. Zhu, H. Bai, and K. Ning, "Network pharmacology databases for traditional Chinese medicine: review and assessment," Frontiers in Pharmacology, vol. 10, p. 123, 2019.

[14] D. Huang, Z. Dong, L. Sun, W. Chen, and L. Sun, "Two neolignans from Penthorum Chinense and their antiproliferative activities," Natural Product Research, vol. 34, no. 11, pp. 1515-1520, 2020.

[15] J. Deng, Y. Ma, Y. He et al., "A network pharmacology-based investigation to the pharmacodynamic material basis and mechanisms of the anti-inflammatory and anti-viral effect of isatis indigotica," Drug Design, Development and Therapy, vol. 15, pp. 3193-3206, 2021.

[16] L. Wang, Y. Ma, Y. He et al., "Systematic investigation of the pharmacological mechanism of Tanreqing injection in treating respiratory diseases by UHPLC/Q-TOF-MS/MS based on multiple in-house chemical libraries coupled with network pharmacology," Journal of Pharmaceutical and Biomedical Analysis, vol. 202, p. 114141, 2021.

[17] C. Xie, P. Zhou, X. Li, and J. Chen, "Research progress on chemical constituents, pharmacological effects, and clinical application of Tripterygium hypoglaucum," Chinese Traditional and Herbal Drugs, vol. 46, no. 13, pp. 1996-2010, 2015.

[18] H. Luo, X. Wu, H. Huang et al., "Simultaneous determination of triptolide, tripterifordin, celastrol and nine sesquiterpene alkaloids in Tripterygium preparations using high-performance liquid chromatography-triple quadrupole mass spectrometry," Journal of Pharmaceutical and Biomedical Analysis, vol. 117, pp. 195-204, 2016.

[19] D. Zhang, L. Qu, Z. Wang, and J. Zhang, "Identification of the chemical components and metabolites of tripterygium glycoside tablets in mice by HPLC-Q/TOF MS," Journal of Chromatography B, vol. 1125, p. 121721, 2019.

[20] Z. Q. Zhou, H. X. Fan, R. R. He et al., "Lycibarbarspermidines A-O, new dicaffeoylspermidine derivatives from wolfberry, with activities against alzheimer's disease and oxidation," Journal of Agricultural and Food Chemistry, vol. 64, no. 11, pp. 2223-2237, 2016. 
[21] X. Xiao, W. Ren, N. Zhang et al., "Comparative study of the chemical constituents and bioactivities of the extracts from fruits, leaves and root barks of lycium barbarum," Molecules (Basel, Switzerland), vol. 24, no. 8, 2019.

[22] L. Qu, Y. Xiao, Z. Jia et al., "Comprehensive two-dimensional liquid chromatography coupled with quadrupole time-offlight mass spectrometry for chemical constituents analysis of tripterygium glycosides tablets," Journal of Chromatography A, vol. 1400, pp. 65-73, 2015.

[23] H. Duan, Y. Takaishi, H. Momota et al., "Immunosuppressive sesquiterpene alkaloids from Tripterygium wilfordii," Journal of Natural Products, vol. 64, no. 5, pp. 582-587, 2001.

[24] M. Li, J. Hou, D. Wei, J. Jiang, M. Yan, and H. Wang, "Identification of major constituents from process residue of tripterygium glycosides by HPLC-QTOF-MS," Journal of China Pharmaceutical University, vol. 46, no. 6, pp. 689-695, 2015.

[25] J. Liu, S. H. Luo, R. Zhang, Z. C. Shu, R.-K. Yang, and J. Yan, "Changes of chemical constituents of tripterygium wilfordii during roasting processing based on UPLC-Q-TOF/MS," Journal of Chinese Mass Spectrometry Society, vol. 39, no. 5, pp. 573-582, 2018.

[26] C. M. Wu, L. M. Zhou, Y. F. Chai, Y. T. Wu, and G. R. Fan, "Three new sesquiterpene alkaloids from the root of Tripterygium wilfordii," Chinese Chemical Letters, vol. 21, no. 7, pp. 830-833, 2010.

[27] H. Duan, Y. Takaishi, Y. Imakura et al., "Sesquiterpene alkaloids from tripterygium hypoglaucum and Tripterygium wilfordii: a new class of potent anti-HIV agents," Journal of Natural Products, vol. 63, no. 3, pp. 357-361, 2000.

[28] J. Y. Bae, B. Avula, J. Zhao et al., "Analysis of prenylflavonoids from aerial parts of Epimedium grandiflorum and dietary supplements using HPTLC, UHPLC-PDA and UHPLC-QToF along with chemometric tools to differentiate Epimedium species," Journal of Pharmaceutical and Biomedical Analysis, vol. 177, p. 112843, 2020.

[29] H. Yuan, S. Cao, S. Chen, L. Guo, J. Zheng, and R. Lin, "Analysis of 11 chemical constituents in Epimedium myrianthum Stearn by RRLC-DAD-ESI-MS2," Chinese Journal of Pharmaceutical Analysis, vol. 34, no. 7, pp. 1156-1160, 2014.

[30] W. Ma, G. Yao, Q. Jia, H. Ouyang, Y. Chang, and J. He, "Qualitative analysis on chemical constituents from Epimedium brevicornu by UPLC-Q-TOF-MS/MS," Journal of Chinese Medicinal Materials, vol. 42, no. 7, pp. 1554-1559, 2019.

[31] L. Lu, H. Zhang, L. Zhao, J. Jia, X. Y. Li, and G. Zhang, "RRLCTOF/MS in rapid identification of 43 chemical constituents of epimedium," Academic Journal of Second Military Medical University, vol. 31, no. 3, pp. 306-310, 2011.

[32] J. Ma, M. Dey, H. Yang et al., "Anti-inflammatory and immunosuppressive compounds from Tripterygium wilfordii," Phytochemistry, vol. 68, no. 8, pp. 1172-1178, 2007.

[33] F. Wang, Y. Zhang, and Y. Zhao, "Chemical constituents of Tripterygium hypoglaucum," Chinese Traditional and Herbal Drugs, vol. 42, no. 1, pp. 46-49, 2011.

[34] L. Zhang, Z. Jiang, T. Tanaka, and I. Kouno, "Studies on tannins from Tripterygium hypoglaucum (lévl.) Hutch," Journal of Chinese Medicinal Materials, vol. 23, no. 9, 1998.

[35] C. A. Lipinski, F. Lombardo, B. W. Dominy, and P. J. Feeney, "Experimental and computational approaches to estimate solubility and permeability in drug discovery and development settings," Advanced Drug Delivery Reviews, vol. 46, no. 1-3, pp. 3-26, 2001.
[36] J. Huang, F. Cheung, H. Y. Tan et al., "Identification of the active compounds and significant pathways of yinchenhao decoction based on network pharmacology," Molecular Medicine Reports, vol. 16, no. 4, pp. 4583-4592, 2017.

[37] A. Daina, O. Michielin, and V. Zoete, "SwissADME: a free web tool to evaluate pharmacokinetics, drug-likeness and medicinal chemistry friendliness of small molecules," Scientific Reports, vol. 7, no. 1, p. 42717, 2017.

[38] R. Ziadlou, A. Barbero, I. Martin et al., "Anti-inflammatory and chondroprotective effects of vanillic acid and epimedin C in human osteoarthritic chondrocytes," Biomolecules, vol. 10, no. 6, 2020.

[39] H. H. Xiao, C. Y. Fung, S. K. Mok et al., "Flavonoids from Herba epimedii selectively activate estrogen receptor alpha $(\mathrm{ER} \alpha)$ and stimulate ER-dependent osteoblastic functions in UMR-106 cells," The Journal of Steroid Biochemistry and Molecular Biology, vol. 143, pp. 141-151, 2014.

[40] L. Zhang, T. Wang, B. S. Zhao et al., "Effect of 2"-O-rhamnosyl icariside II, baohuoside I and baohuoside II in herba epimedii on cytotoxicity indices in HL-7702 and HepG2 cells," Molecules, vol. 24, no. 7, 2019.

[41] Z. Jing, Z. Z. Ma, D. D. Zhang, and Y. Y. Shi, "Exploration of component-target-pathway of Kunxian capsules in treatment of proteinuria," Chinese Journal of Hospital Pharmacy, vol. 40, no. 23, pp. 2396-2405, 2020.

[42] Y. Zhang, X. Mao, W. Li et al., "Tripterygium wilfordii: an inspiring resource for rheumatoid arthritis treatment," $M e$ dicinal Research Reviews, vol. 41, no. 3, pp. 1337-1374, 2021.

[43] J. Cui, X. Li, S. Wang et al., "Triptolide prevents bone loss via suppressing osteoclastogenesis through inhibiting PI3KAKT-NFATc1 pathway," Journal of Cellular and Molecular Medicine, vol. 24, no. 11, pp. 6149-6161, 2020.

[44] D. Fan, X. He, Y. Bian et al., "Triptolide modulates TREM-1 signal pathway to inhibit the inflammatory response in rheumatoid arthritis," International Journal of Molecular Sciences, vol. 17, no. 4, p. 498, 2016.

[45] Y. Yang, Y. Ye, Q. Qiu et al., “Triptolide inhibits the migration and invasion of rheumatoid fibroblast-like synoviocytes by blocking the activation of the JNK MAPK pathway," International Immunopharmacology, vol. 41, pp. 8-16, 2016.

[46] R. Ganesan and M. Rasool, "Fibroblast-like synoviocytes-dependent effector molecules as a critical mediator for rheumatoid arthritis: current status and future directions," International Reviews of Immunology, vol. 36, no. 1, pp. 20-30, 2017.

[47] S. Li, J. W. Chen, X. Xie et al., "Autophagy inhibitor regulates apoptosis and proliferation of synovial fibroblasts through the inhibition of PI3K/AKT pathway in collagen-induced arthritis rat model," American Journal of Tourism Research, vol. 9, no. 5, pp. 2065-2076, 2017.

[48] E. Karouzakis, R. E. Gay, S. Gay, and M. Neidhart, "Epigenetic control in rheumatoid arthritis synovial fibroblasts," Nature Reviews Rheumatology, vol. 5, no. 5, pp. 266-272, 2009.

[49] X. Li, W. Wei, Z. Zhao, and S. Lv, "Tripterine up-regulates miR223 to alleviate lipopolysaccharide-induced damage in murine chondrogenic ATDC5 cells," International Journal of Immunopathology and Pharmacology, vol. 33, p. 2058738418824521, 2019.

[50] V. K. W. Wong, C. Qiu, S. W. Xu et al., "Ca 2+ signalling plays a role in celastrol-mediated suppression of synovial fibroblasts of rheumatoid arthritis patients and experimental arthritis in rats," British Journal of Pharmacology, vol. 176, no. 16, pp. 2922-2944, 2019.

[51] L. Chi, W. Gao, X. Shu, and X. Lu, "A natural flavonoid glucoside, icariin, regulates Th17 and alleviates rheumatoid 
arthritis in a murine model," Mediators of Inflammation, vol. 2014, p. 392062, 2014.

[52] Z.-M. Wu, J. Luo, X.-D. Shi, S.-X. Zhang, X.-B. Zhu, and J. Guo, "Icariin alleviates rheumatoid arthritis via regulating miR-223-3p/NLRP3 signalling axis," Autoimmunity, vol. 53, no. 8, pp. 450-458, 2020.

[53] R. Ziadlou, A. Barbero, M. J. Stoddart et al., "Regulation of inflammatory response in human osteoarthritic chondrocytes by novel herbal small molecules," International Journal of Molecular Sciences, vol. 20, no. 22, 2019.

[54] Y. K. Jung, Y. M. Kang, and S. Han, "Osteoclasts in the inflammatory arthritis: implications for pathologic osteolysis," Immune network, vol. 19, no. 1, p. e2, 2019.

[55] Y. Tang, Q. Liu, Y. Feng et al., "Tripterygium ingredients for pathogenicity cells in rheumatoid arthritis," Frontiers in Pharmacology, vol. 11, p. 583171, 2020. 